\title{
Trauma-related rumination mediates the effect of naturally occurring depressive symptoms but not momentary low mood on trauma intrusions
}

\author{
Rie Kubota, Reginald D.V. Nixon, and Junwen Chen \\ School of Psychology, Flinders University, Adelaide, South Australia, Australia
}

\begin{abstract}
Comorbid depression is known to contribute to the maintenance of posttraumatic stress disorder (PTSD) including distressing intrusive trauma memories. It is theorised that depression is a risk factor for persistent PTSD through preventing optimal habituation of distress provoked by trauma memories and reminders, but the underlying cognitive mechanisms responsible are uncertain. The present study investigated trauma-related rumination as a possible mediator for the effect of depression on trauma intrusions. Participants received a low mood induction or control procedure. Following viewing an analogue trauma film, frequency of film-related intrusions and associated distress levels were measured and at l-week follow-up. Between the two occasions, participants rated their levels of rumination about the film. Existing depression symptoms but not induced momentary sad mood predicted frequency of film intrusions and associated distress at l-week follow-up. Some evidence was found that ruminative trauma processing mediated the relationship between baseline depressive symptoms and later intrusion frequency and associated distress. Future research is warranted to better understand the role of rumination in the depression-intrusion relationship, which may shed light on the clinical applicability of rumination-targeted intervention for PTSD and comorbid depression.
\end{abstract}

Key words: comorbid depression, intrusion, posttraumatic stress disorder, rumination, trauma

Posttraumatic stress disorder (PTSD) is often comorbid with clinical depression with average reported prevalence rates of approximately $50 \%$ for major depressive disorder (e.g., Blanchard, Buckley, Hickling, \& Taylor, 1998; Kessler, Sonnega, Bromet, Hughes, \& Nelson, 1995). According to emotional processing theory, depression contributes to suboptimal recovery from PTSD by reducing one's capacity for habituation of anxiety to the trauma memory and cues, resulting in less successful new (adaptive) learning (Foa \& Kozak, 1986). The theory further posits several reasons for the negative impact of depression, including: excessively diminished reactivity or increased responsiveness to fear, reduced distress intolerance, heightened self-perceptions of ineffectiveness or helplessness, and unhelpful beliefs about ability to cope with fear (Foa \& Kozak, 1986). In clinical research, prior depression has been shown to increase the risk of developing PTSD upon trauma exposure (Breslau, Davis, Peterson, \& Schultz, 1997, 2000; Shalev et al., 1998) and a trend for those with comorbid depression to be more

Correspondence: Reginald D.V. Nixon, PhD, School of Psychology, Flinders University, GPO Box 2100, Adelaide, SA 5001, Australia. Email: reg.nixon@flinders.edu.au

Received 11 October 2013. Accepted for publication 10 July 2014. (C) 2014 The Australian Psychological Society likely to retain their PTSD diagnosis over time (Shalev et al., 1998). Comorbid depression cases are also associated with greater PTSD symptom severity (Nixon, Resick, \& Nishith, 2004). In relation to intrusive distressing recollection of trauma, the hallmark symptom of PTSD (American Psychiatric Association, 2013), severity of depression has been shown to positively correlate with frequency of intrusions (Brewin, Hunter, Carroll, \& Tata, 1996; Kuyken \& Brewin, 1994). However, little is known about the mechanisms by which depression might lead to the persistence of PTSD, or of relevance to the present study, distressing posttrauma intrusions.

According to information processing theories of PTSD, the persistence of intrusive re-experiencing (and other symptoms) occurs as a result of dysfunctional processing of the traumatic event and/or its consequences (e.g., Brewin, Dalgleish, \& Joseph, 1996; Ehlers \& Clark, 2000; Foa \& Kozak, 1986). Further, the theories share a view that certain cognitive styles make processing more dysfunctional. Such styles may reflect strategies employed by victims of a traumatic event to attempt to reduce intrusive re-experiencing and associated emotional distress, and may represent an emotion regulation strategy (Ehlers \& Steil, 1995). Accordingly, because depression maintains posttrauma intrusions, as argued below, it is reasonable to expect that certain 
cognitive coping strategies employed by individuals with depression enhance dysfunctional information processing of the trauma memory, thereby resulting in persistent trauma intrusions.

One candidate dysfunctional cognitive strategy that may explain how depression affects posttrauma intrusion is rumination, commonly defined as repetitive thinking about past negative experiences and negative mood (Nolen-Hoeksema, 1991; Nolen-Hoeksema, Wisco, \& Lyubomirsky, 2008). Trauma-related rumination in PTSD sufferers is a common cognitive strategy in response to and to make sense of the causes and consequences of the trauma (Ehlers \& Clark, 2000), and levels of trauma-related rumination are thought of as a marker of PTSD symptom severity (Ehlers \& Steil, 1995). However, the degree to which it is maladaptive differs depending on the way individuals ruminate (Watkins, 2008). Rumination is commonly classified into two types depending on its processes and consequences, namely reflective pondering and brooding. While reflective pondering is relatively adaptive as it is seen as a method likely to lead to finding solutions to a troubling situation (Martin \& Tesser, 1996), brooding is considered maladaptive due to a continual focus on negative or emotional aspects of the situation without focusing on features of the situation or behaviours that are amenable to change (Joormann, Dkane, \& Gotlib, 2006). Of these types, it is the brooding rumination that characterises cognitive response to negative events typically employed by people with depression (see Ehring $\&$ Watkins, 2008; Nolen-Hoeksema et al., 2008, for review). It thus follows that depression may be a risk factor for persistence of PTSD via facilitation of maladaptive rumination, assuming that this type of rumination does have a detrimental effect on posttrauma adjustment.

Theoretically, the harmful effect of rumination on PTSD/ intrusions has been speculated by the cognitive model of PTSD (Ehlers \& Clark, 2000; Ehlers \& Steil, 1995). First, rumination serves as a form of cognitive avoidance, interfering with the formation of a more complete trauma memory. Dwelling on negative issues related to the trauma but not thinking actively about the traumatic experience itself hinders the emotional processing of the trauma (Ehring, Fuchs, \& Klasener, 2009; Foa \& Kozak, 1986). This proposal is in line with a finding that occurrence of 'why' and 'what if' type questions by trauma survivors predicted later PTSD severity (Michael, Halligan, Clark, \& Ehlers, 2007). Second, rumination strengthens problematic appraisals of trauma and its meaning, and prevents them from being replaced with more adaptive appraisals. This suggestion corresponds with empirical findings that rumination is associated with more negative appraisals of situations, and a tendency to attend to and remember negative information rather than positive information (see Nolen-Hoeksema et al., 2008, for review). Finally, rumination provides internal retrieval cues for memories of a traumatic event, which in turn increases intrusions. This proposal is in agreement with a finding that in PTSD sufferers, rumination was a strong trigger for intrusions of a trauma scene (Birrer, Michael, \& Munsch, 2007).

In line with the cognitive model (Ehlers \& Clark, 2000), clinical studies have shown that frequency of trauma-related rumination was cross-sectionally correlated with PTSD symptom severity (Evans, Ehlers, Mezey, \& Clark, 2007; Steil $\delta$ Ehlers, 2000). Further, prospective research of trauma survivors consistently found that frequency of rumination was a predictor of PTSD symptom severity with medium to large effect sizes (e.g., Ehlers, Mayou, \& Bryant, 1998; Murray, Ehlers, \& Mayou, 2002). In contrast, direct manipulations of rumination yielded somewhat mixed findings. In Ball and Brewin (2012), moderate-high trait ruminators who were instructed to ruminate in a maladaptive manner after seeing a film of road traffic accidents later reported a greater number of film-related intrusive memories than no-task controls, suggesting a causal effect of rumination on intrusive memories. However, other studies (Ehring, Szeimies, \& Schaffrick, 2009; Zetsche, Ehring, \& Ehlers, 2009) found with unselected participants that ruminating about an analogue traumatic film clip in the same manner subsequently produced comparable numbers of film-related intrusions as comparison participants.

As speculated by Ball and Brewin (2012), the inconsistent findings might reflect that only individuals with existing ruminative tendency have an ability to engage in posttrauma ruminative thinking on command in a way that produces greater posttrauma intrusions. Therefore, people with trait ruminative thinking style may be at higher risk of developing intrusions upon trauma exposure than non-habitual ruminators. As trait ruminative tendency is known to contribute to greater depression severity (e.g., Nolen-Hoeksema et al., 2008), high levels of depression can be seen as a risk factor for the maintenance of posttrauma intrusions through its easily activated ruminative mode of thinking. From this perspective, the mechanism for the depression-intrusion relationship can be seen as being the relatively easily triggered rumination after trauma exposure maintaining posttraumatic intrusions in individuals with depression (who are likely to already be habitual ruminators).

Independent of trait depression and ruminative tendency, the mood-as-input model of pathological repetitive thinking (Meeten \& Davey, 2011) offers an explanation of how temporary low mood also causes rumination. Experimental methods of mood induction have been widely used to test a causal effect of emotions on cognitions (Eich, Ng, Macaulay, Percy, \& Grebneva, 2007). Hawksley and Davey (2010) manipulated mood (negative or positive) and stop rule ('as many as can' or 'feel like continuing') in unselected participants. During a rumination interview, participants were 
asked to think back to and respond to questions regarding a past depressing experience. At this time, those in the as many as can' group were instructed to participate in the interview until they had completed exploring their depression, while those in the other condition were asked to remain so long as they felt like continuing. The study found that persistence at the interview was greatest in the low mood group adopting 'as many as can' stop rule, suggesting that induced transient low mood during ruminative mode of processing caused the maintenance of rumination about negative experiences. This has not been tested in the context of trauma memory.

The effect of temporary low mood has also been found in relation to posttrauma intrusions. Wilksch (2011) had participants to watch an analogue trauma film clip and then randomly allocated them to either sad mood or control condition. In this sample, film-related intrusions in the sad mood condition reduced at a slower rate over a week compared with controls, suggesting a causal role of induced momentary sad mood in the maintenance of distressing trauma intrusions.

Taken together, depression and low mood appear to be risk factors for the persistence of distressing trauma intrusions, and as argued, trauma-related rumination is likely to play an important role in this relationship. As discussed earlier, emotional processing theory (Foa \& Kozak, 1986) posits a role for depression to interfere with habituation of anxiety in response to trauma memory and cues but the reasons for this do not explicitly include the role of rumination. While Ehlers and Clark (2000) outline the role of rumination in the persistence of PTSD, how this might be influenced by low mood or depressed symptoms is not explained.

Utilising a film clip of fictional traumatic contents, the current study sought to test the effect of induced low mood and baseline depressive symptoms on trauma intrusions and the mediating role of trauma-related rumination in the relationship between low mood/baseline depressive symptoms and trauma intrusions. We employed two induced low mood groups that were compared with the control group; in one group, low mood was induced before viewing the film because past research have shown that depression prior to trauma exposure is a risk factor for the development of PTSD (e.g., Breslau et al., 2000), implying a possibility that low mood during trauma exposure prevents optimal processing; the other group received the induction after viewing the film given comorbid depression may contribute to the maintenance of PTSD (Shalev et al., 1998), implying low mood may affect posttrauma processing. Participants' frequency of filmrelated intrusions and associated distress levels were measured during 5-min monitoring tasks within the experimental session as well as at 1 -week follow-up. Levels of film-related rumination were measured during the l-week interval. In line with past research (e.g., Ball \& Brewin, 2012; Ehring,
Szeimies, et al., 2009), both intrusion frequency and intrusion-related distress were measured. While frequency of intrusions is commonly examined in analogue trauma intrusions research (Holmes \& Bourne, 2008), some researchers suggest that it is particularly those intrusions that are experienced as distressing that contribute to the persistence of PTSD (Steil \& Ehlers, 2000). Participants' levels of trait ruminative tendency were also measured given its close relationship with depression (e.g., Nolen-Hoeksema et al., 2008) and with ruminative response to analogue trauma (Ball \& Brewin, 2012).

We had three main hypotheses. First, we predicted that participants with higher baseline depressive symptom levels would report more frequent intrusions of the trauma film and greater associated distress at 1-week follow-up than those with lower levels of depressive symptoms. Second, participants who underwent a low mood induction (before or after the film) would experience slower reductions in their intrusions and associated distress over a week interval than the control participants. Third, we predicted that trauma-related rumination would mediate the relationship between baseline depression/induced sad mood and trauma intrusions and associated distress such that greater baseline depression symptoms/induced sad mood would lead to greater trauma-related rumination during the week following viewing the trauma film, which in turn would lead to more frequent and more distressing trauma intrusions at l-week follow-up. We also expected that induced low mood (before or after the film) would increase levels of traumarelated rumination over a l-week interval, and that baseline levels of depression would be positively correlated with levels of trauma-related rumination, and general (trait) ruminative style.

\section{METHOD}

\section{Participants}

Participants were 90 university students. Individuals who reported having a formal diagnosis of a mental health disorder (e.g., PTSD, depression, anxiety) were excluded. The sample was predominantly female $(67.8 \%)$ with a mean age of 23.93 years (standard deviation $=6.86$ ). Participants received course credits or financial reimbursement (\$AUD20).

\section{Materials}

\section{Trauma film}

Participants viewed an 8-min clip from the fictional French film Irreversible, which contained graphic scenes of physical and sexual violence. Previous experimental studies (e.g., 
Nixon, Nehmy, \& Seymour, 2007; Wilksch, 2011) had used the same film to successfully induce intrusive experiences, with trauma films an established method of studying intrusive phenomenon (Holmes \& Bourne, 2008).

\section{Mood induction}

In the sad mood conditions, participants wrote down details about a time in their life when they had felt sad, and then listened to orchestral music of a classical genre ('Russia under the Mongolian Yoke' from 'Alexander Nevsky' by Sergei Prokofiev) while they continued to think about the event that they had just written. The music piece has been shown effective in inducing sad mood (Werner-Seidler $\delta$ Moulds, 2011). This procedure which combined both sad mood-suggestive music and the autobiographical recall of a sad mood-evoking event has been used successfully to induce transient low mood (e.g., Eich et al., 2007; Werner-Seidler \& Moulds, 2011). The control group was utilised to mitigate the possible effect of time and cognitive load associated with the mood induction procedure on processing of film-related memory. Specifically, participants in the control condition went through the same procedure as the sad mood groups but listened to another piece from 'Alexander Nevsky' by Sergei Prokofiev, and wrote about preparing and eating their typical dinner. The music piece was found to be neutral, relative to the sad music, as assessed by mood ratings (M. L. Moulds, personal communication, September 30, 2013). Participants reported how much they felt sad on a rating scale ranging from 1 (not at all sad) to 10 (extremely sad) after the low mood induction or control task.

\section{Measures}

\section{Film-related intrusions}

Intrusive memory from the film clip was measured in 5-min intrusion monitoring tasks. Participants were asked to think about anything they wished but to indicate each time they had a spontaneous visual image from the film by raising their finger. The frequency of the intrusions was recorded by the researcher. The 5-min monitoring intervals were conducted after the trauma film (T1), after the mood manipulation (or filler task) (T2), and at 1-week follow-up (T3). Participants also rated how distressed they felt from the memories using a single-item scale (where $1=$ not at all to $10=$ extremely distressed .

\section{Trait rumination and film-related rumination}

The Perseverative Thinking Questionnaire (PTQ; Ehring, 2007) was administered at baseline to measure participants' trait levels of maladaptive rumination. Participants rated each of 15 items on a scale ranging from 0 (never) to 4 (almost always). The PTQ has good internal consistency with Cronbach's $\alpha$ ranging between .94 and .95 depending on samples (Ehring etal., 2011). In the present study, Cronbach's $\alpha=.96$.

The modified State version of the Perseverative Thinking Questionnaire (PTQ-S) was used to measure the levels of maladaptive rumination indexed to the film-related memory. Internal consistency (Cronbach's $\alpha$ ) is reported to be .89 (Zetsche et al., 2009). In the present study, Cronbach's $\alpha s=.96$ (day 3) and .97 (day 7).

\section{Baseline depression symptoms}

The Depression subscale of the Depression Anxiety Stress Scale (DASS-D; Lovibond \& Lovibond, 1995), comprising 14 items, was used to measure an individual's baseline depression symptoms. Participants responded according to how much the statement applied to them over the past week using a 4-point severity scale. The DASS-D possesses excellent internal consistency with Cronbach's $\alpha=.96$ (Brown, Chorpita, Korotitsch, \& Barlow, 1997). In the present study, Cronbach's $\alpha=.94$.

\section{Prior trauma}

The following well-established measures relating to prior trauma were administered at baseline in the event that these variables needed to be controlled in subsequent analyses. The PTSD Checklist (PCL; Weathers, Litz, Herman, Huska, \& Keane, 1993) is a 17-item self-report checklist of PTSD symptoms. Participants who reported that they had been exposed to 'trauma' answered how often they had been bothered by each symptom in the previous month on a 5-point severity scale. The Posttraumatic Cognitions Inventory (PTCI; Foa, Ehlers, Clark, Tolin, \& Orsillo, 1999) is a 36-item instrument used to assess participants' baseline trauma-related thoughts and appraisals, specifically, negative cognitions about the self, negative cognitions about the world, and self-blame. Participants rated each item on a 7-point scale ranging from 1 (totally disagree) to 7 (totally agree).

\section{Procedure}

Figure 1 exhibits a flow diagram summarising experimental method and procedures. Following completion of the baseline questionnaires (PCL, PTCI, DASS-D, and PTQ) and mood rating, participants were randomly allocated to either the sad mood induced before the film (sad before film $(\mathrm{SBF})$ ), sad mood induced after the film (sad after film (SAF)), or control condition. Before viewing, the film participants in the SBF condition received sad mood induction, after which they re-rated their mood and were then shown the film. The SAF and control groups did not go through 


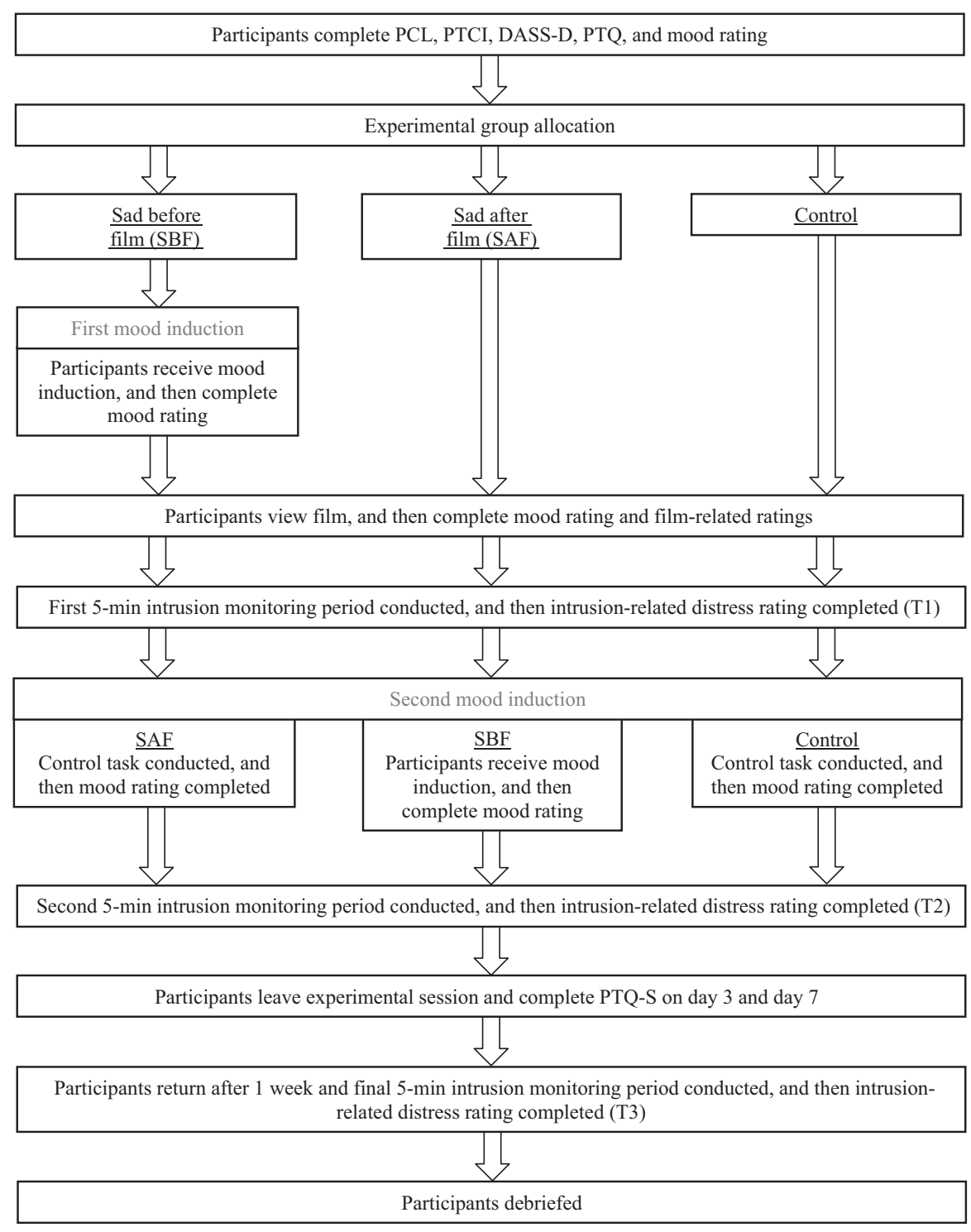

Figure 1 Flow diagram summarising experimental method and procedures. PCL = PTSD Checklist; PTCI = Posttraumatic Cognitions Inventory; DASS-D = Depression subscale of the Depression Anxiety Stress Scale; PTQ = Perseverative Thinking Questionnaire; PTQ-S = State version of the Perseverative Thinking Questionnaire.

the mood induction/control procedure at this stage. Following the film, all participants indicated how unpleasant and distressing the film was, how much attention they paid to the film, and rated their current mood. All participants then completed an intrusion monitoring task and rated their intrusion-related distress (T1). Next, the SAF and control groups received the sad mood induction or control tasks, while the SBF group completed a filler task utilising the control procedure. All participants again completed the intrusion monitoring task and rated their intrusion-related distress (T2). Participants completed the PTQ-S on the 3rd and 7th day following the experimental session. At l-week follow-up, participants completed the final intrusion monitoring task and distress rating (T3).

\section{RESULTS}

A total of 81 participants completed both the initial and follow-up sessions with 26, 26, and 29 in the SBF, SAF, and control groups, respectively. Nine participants were not included in analyses (either did not watch entire film clip or did not complete follow-up). The groups did not significantly differ in age, $F(2,78)=0.82, p=.45, \eta_{\mathrm{p}}{ }^{2}=.02$; gender compositions, $\chi^{2}(2, N=81)=.31, p=.86, \varphi_{c}=.06$; unpleasantness of the film, $F(2,78)=0.76, p=.47, \eta_{\mathrm{p}}{ }^{2}=.02$; perceived distressing levels of the film, $F(2,78)=0.42, p=.66$, $\eta_{\mathrm{p}}{ }^{2}=.01$; and levels of attention paid to the film, $F(2,78)=0.89, p=.42, \eta_{\mathrm{p}}^{2}=.02$. The groups were not significantly different on the DASS-D, $F(2,78)=1.20$, 
Table 1 Demographic characteristics, film ratings, and baseline questionnaire scores by group

\begin{tabular}{|c|c|c|c|c|c|c|}
\hline & \multicolumn{2}{|c|}{$\begin{array}{c}\text { Sad before film } \\
(n=26)\end{array}$} & \multicolumn{2}{|c|}{$\begin{array}{l}\text { Sad after film } \\
\quad(n=26)\end{array}$} & \multicolumn{2}{|c|}{$\begin{array}{l}\text { Control } \\
(n=29)\end{array}$} \\
\hline & $M($ or $n)$ & $S D($ or $\%)$ & $M($ or $n)$ & $S D($ or $\%)$ & $M($ or $n)$ & $S D($ or $\%)$ \\
\hline \multicolumn{7}{|l|}{ Demographics } \\
\hline Age & 25.15 & 8.11 & 23.25 & 4.93 & 23.10 & 5.78 \\
\hline Female & 17 & $65 \%$ & 18 & $69 \%$ & 18 & $62 \%$ \\
\hline \multicolumn{7}{|l|}{ Film ratings } \\
\hline Distressing & 7.81 & 1.36 & 8.27 & 1.80 & 7.93 & 2.33 \\
\hline Unpleasantness & 8.50 & 1.45 & 9.00 & 1.20 & 8.83 & 1.73 \\
\hline Attention & 7.81 & 1.20 & 7.62 & 1.53 & 8.10 & 1.37 \\
\hline \multicolumn{7}{|c|}{ Baseline questionnaires } \\
\hline DASS-D & 4.54 & 4.98 & 5.35 & 6.60 & 7.28 & 8.14 \\
\hline PTQ & 25.46 & 10.77 & 22.15 & 13.92 & 28.03 & 13.97 \\
\hline & \multicolumn{2}{|c|}{$(n=20)$} & \multicolumn{2}{|c|}{$(n=18)$} & \multicolumn{2}{|c|}{$(n=22)$} \\
\hline PCL & 29.65 & 12.59 & 24.50 & 10.17 & 30.45 & 12.28 \\
\hline PTCI & 82.85 & 27.94 & 80.28 & 37.00 & 84.82 & 37.82 \\
\hline
\end{tabular}

Note. DASS-D = Depression subscale of the Depression Anxiety Stress Scale; PCL = PTSD Checklist; PTCI = Posttraumatic Cognitions Inventory; PTQ $=$ Perseverative Thinking Questionnaire.

Table 2 Correlations between baseline PTSD symptoms and beliefs, and intrusion and rumination variables

\begin{tabular}{|c|c|c|}
\hline & $\begin{array}{l}\text { PCL } \\
r\end{array}$ & $\begin{array}{l}\text { PTCI } \\
r\end{array}$ \\
\hline & \multicolumn{2}{|c|}{ Tl (immediately after film) } \\
\hline Intrusion frequency & .09 & .21 \\
\hline \multirow[t]{2}{*}{ Intrusion-related distress } & $.26^{*}$ & .18 \\
\hline & \multicolumn{2}{|c|}{$\begin{array}{l}\text { T2 (following mood induction for } \\
\text { SAF/filler task for SBF and control) }\end{array}$} \\
\hline Intrusion frequency & .09 & .24 \\
\hline \multirow[t]{2}{*}{ Intrusion-related distress } & $.28^{*}$ & $.30^{*}$ \\
\hline & \multicolumn{2}{|c|}{ 1-week interval } \\
\hline PTQ-S Day 3 & .17 & .23 \\
\hline \multirow{2}{*}{ PTQ-S Day 7} & .02 & .12 \\
\hline & \multicolumn{2}{|c|}{ T3 (follow-up) } \\
\hline Intrusion frequency & .15 & .20 \\
\hline Intrusion-related distress & .21 & .14 \\
\hline
\end{tabular}

Note. $n=60$. PCL $=$ PTSD Checklist; PTCI $=$ Posttraumatic Cognitions Inventory; PTQ-S = State version of the Perseverative Thinking Questionnaire.

${ }^{*} p<.05$.

$p=.31, \eta_{\mathrm{p}}{ }^{2}=.03$, and PTQ, $F(2,78)=1.40, p=.25, \eta_{\mathrm{p}}{ }^{2}=.04$ The groups did not significantly vary on whether they reported any experience of prior trauma, $\chi^{2}(2, N=81)=.48$, $p=.79, \varphi_{\mathrm{c}}=.07 ; \mathrm{PCL}, F(2,57)=1.43, p=.25, \eta_{\mathrm{p}}^{2}=.05 ;$ and PTCI, $F(2,57)=0.09, p=.92, \eta_{\mathrm{p}}{ }^{2}=.003$. Descriptive data are reported in Table 1 . The PCL and PTCI did not significantly correlate with the dependent variables of interest consistently across time and were not considered further (see Table 2 for correlations).

\section{Mood manipulation check}

Table 3 summarises the descriptive data for sad mood ratings by group and time. At baseline, a one-way analysis of variance (ANOVA) showed the groups were not significantly different from each other in their sadness ratings,
$F(2,78)=0.43, p=.65, \eta_{\mathrm{p}}{ }^{2}=.11$. Manipulation checks were conducted separately for the two sad groups due to the different timings of mood induction for the groups. For the SBF group, the increase in sadness scores following induction was significant, $t(25)=6.35, p<.001$, with a mean difference of 2.04 (95\% confidence interval (CI) 1.38, 2.70), $d=1.82$. For the SAF group, the change in sadness scores from pre- to post-manipulation was compared against the control condition with a mixed ANOVA that revealed a significant group $\times$ time interaction on sadness ratings, $F(1,53)=4.97, p=.03, \eta_{\mathrm{p}}{ }^{2}=.09$. At post-manipulation, the SAF group rated significantly higher on the sad mood rating than the control group, $t(53)=3.19, p<.01$, with a mean difference of 2.04 (95\% CI $0.76,3.32), d=0.86$. Thus, the sad mood manipulation appeared to have been effective.

\section{Main results}

\section{Effects of induced sad mood}

Table 4 summarises the descriptive data for intrusion frequency, intrusion-related distress, and film-related rumination (PTQ-S) over the course of the experiment. Three (group $(\mathrm{SBF}, \mathrm{SAF}$, control) $) \times 3($ time $(\mathrm{T} 1, \mathrm{~T} 2, \mathrm{~T} 3))$ mixed ANOVAs were conducted to examine reduction of intrusions and associated distress over the l-week interval. Contrary to prediction, the reduction in intrusion frequency in the two sad conditions over time was not significantly slower compared with the control condition, $F(3.34,130.06)=0.56$, $p=.66, \eta_{\mathrm{p}}{ }^{2}=.01$. This null result remained unchanged when the two sad groups (no significant difference between them, $\left.F(1.59,76.66)=0.97, p=.37, \eta_{\mathrm{p}}^{2}=.02\right)$ were collapsed together to be compared with the control group, $F(1.64$, $129.34)=0.07, p=.90, \eta_{p}^{2}=.001$. The same pattern of findings across the three groups was observed when 
Table 3 Sad mood ratings by group and time

\begin{tabular}{|c|c|c|c|c|c|c|}
\hline & \multicolumn{2}{|c|}{ Sad before film $(n=26)$} & \multicolumn{2}{|c|}{ Sad after film $(n=26)$} & \multicolumn{2}{|c|}{ Control $(n=29)$} \\
\hline & $M$ & $S D$ & $M$ & $S D$ & $M$ & $S D$ \\
\hline Baseline & 2.27 & 1.37 & 2.69 & 1.81 & 2.45 & 1.72 \\
\hline First mood induction & 4.31 & 1.85 & - & - & - & - \\
\hline Post-film & 5.00 & 2.28 & 5.15 & 2.57 & 4.69 & 2.98 \\
\hline Second mood induction & 3.27 & 1.87 & 5.38 & 2.32 & 3.34 & 2.41 \\
\hline Follow-up & 1.73 & 0.87 & 2.08 & 1.67 & 2.28 & 1.87 \\
\hline
\end{tabular}

Note. $\mathrm{SAF}=$ sad after film; $\mathrm{SBF}=$ sad before film.

Table 4 Intrusion and rumination variables by group and time

\begin{tabular}{|c|c|c|c|c|c|c|}
\hline & \multicolumn{2}{|c|}{$\begin{array}{l}\text { Sad before film } \\
\qquad(n=26)\end{array}$} & \multicolumn{2}{|c|}{$\begin{array}{c}\text { Sad after film } \\
\quad(n=26)\end{array}$} & \multicolumn{2}{|c|}{$\begin{array}{l}\text { Control } \\
(n=29)\end{array}$} \\
\hline & $M(S D)$ & $95 \%$ CI & $M(S D)$ & $95 \% \mathrm{CI}$ & $M(S D)$ & $95 \% \mathrm{CI}$ \\
\hline & \multicolumn{6}{|c|}{ T1 (immediately after film) } \\
\hline Intrusion frequency & $8.04(7.30)$ & $(5.45,10.63)$ & $10.88(5.29)$ & $(8.29,13.48)$ & $9.90(7.08)$ & $(7.44,12.35)$ \\
\hline Intrusion-related distress & $4.54(2.50)$ & $(3.56,5.52)$ & $5.23(2.39)$ & $(4.25,6.22)$ & $5.17(2.65)$ & $(4.24,6.10)$ \\
\hline $\begin{array}{l}\text { Intrusion frequency } \\
\text { Intrusion-related distress }\end{array}$ & $\begin{array}{ll}4.27 & (4.63) \\
2.89 & (1.73)\end{array}$ & $\begin{array}{l}\text { T2 (following } \\
(2.13,6.41) \\
(2.03,3.74)\end{array}$ & $\begin{array}{l}\text { ood induction fo } \\
6.08(4.77) \\
3.69(2.49)\end{array}$ & $\begin{array}{l}\text { AF/filler task fo } \\
\quad(3.94,8.22) \\
(2.83,4.55)\end{array}$ & $\begin{array}{c}\text { BF and control) } \\
5.93(6.65) \\
3.38(2.29)\end{array}$ & $\begin{array}{l}(3.91,7.96) \\
(2.57,4.19)\end{array}$ \\
\hline $\begin{array}{l}\text { PTQ-S (Day 3) } \\
\text { PTQ-S (Day 7) }\end{array}$ & $\begin{aligned} 13.73 & (10.85) \\
8.81 & (10.25)\end{aligned}$ & $\begin{array}{l}(8.80,18.66) \\
(4.36,13.26)\end{array}$ & $\begin{array}{rr} & 1 \text {-wed } \\
15.96 & (12.54) \\
7.65 & (11.75)\end{array}$ & $\begin{array}{l}\text { interval } \\
(11.03,20.89) \\
(3.20,12.11)\end{array}$ & $\begin{array}{l}18.85(14.09) \\
12.37(12.04)\end{array}$ & $\begin{array}{r}(14.18,23.52) \\
(8.15,16.58)\end{array}$ \\
\hline $\begin{array}{l}\text { Intrusion frequency } \\
\text { Intrusion-related distress }\end{array}$ & $\begin{array}{ll}1.69 & (2.17) \\
1.96(1.18)\end{array}$ & $\begin{array}{l}(0.56,2.88) \\
(1.35,2.58)\end{array}$ & $\begin{array}{rr} & \mathrm{T} 3 \\
2.77 & (2.37) \\
2.04 & (1.34)\end{array}$ & $\begin{array}{l}\text { (1.up) } \\
(1.58,3.96) \\
(1.42,2.66)\end{array}$ & $\begin{array}{ll}3.00(4.06) \\
2.38(2.03)\end{array}$ & $\begin{array}{l}(1.88,4.12) \\
(1.80,2.96)\end{array}$ \\
\hline
\end{tabular}

Note. $\mathrm{CI}=$ confidence interval; $\mathrm{PTQ}-\mathrm{S}=$ State version of the Perseverative Thinking Questionnaire.

intrusion-related distress was examined, $F(3.59$, 139.81) $=0.66, p=.60, \eta_{\mathrm{p}}^{2}=.02$. Again, this remained unchanged when the two combined sad groups (which were not significantly different from each other, $F(1.88$, 93.82) $=1.13, p=.33, \eta_{\mathrm{p}}{ }^{2}=.02$ ) were compared against the control group, $F(1.77,139.96)=0.22, p=.78, \eta_{\mathrm{p}}^{2}=.003$.

In terms of induced mood impacting on trauma-related rumination (PTQ-S), a one-way ANOVA did not reveal significant group differences, $F(2,78)=1.14, p=.33, \eta_{\mathrm{p}}{ }^{2}=.01$ and $F(2,78)=1.29, p=.28, \eta_{\mathrm{p}}{ }^{2}=.01$ for day 3 and day 7 , respectively. The results remained unchanged when two sad mood groups (which did not significantly differ from one another, $F(1,50)=0.47, p=.50, \eta_{\mathrm{p}}{ }^{2}=.01$ and $F(1$, $50)=0.14, p=.71, \eta_{\mathrm{p}}^{2}=.003$ for day 3 and day 7 , respectively) were collapsed together to be compared with the control group, $F(1,79)=1.89, p=.17, \eta_{\mathrm{p}}{ }^{2}=.02$ and $F(1$, $79)=2.48, p=.12, \eta_{\mathrm{p}}{ }^{2}=.03$ for day 3 and day 7 , respectively. Thus, the hypothesis that experimentally induced sad mood would result in greater levels of maladaptive traumarelated rumination and slower reduction of trauma intrusions and associated distress was not supported.

\section{Correlations between baseline depression, trait rumination, trauma-related rumination, and trauma intrusion frequency and associated distress}

Prior to testing of the mediating role of trauma-related rumination, correlation analyses were conducted to examine (c) 2014 The Australian Psychological Society relationships between baseline depression (DASS-D), baseline ruminative tendency (PTQ), trauma-related rumination (PTQ-S) measured on day 3 and day 7, and intrusion frequency and associated distress at the 1-week follow-up (see Table 5 for descriptive statistics and intercorrelations between variables). As predicted, depression symptom levels were positively correlated with trauma-related rumination measured on day 3, frequency of trauma intrusions, and associated distress, with the exception that the correlation between depression and trauma-related rumination measured on day 7 did not reach significance. Consequently, the main analysis of the mediating role of trauma-related rumination for the effect of baseline depression on intrusion frequency and associated distress was conducted using the PTQ-S from day 3 but not day 7. Trait rumination (PTQ) was positively correlated with depression, trauma-related rumination measured on day 3 and day 7, and frequency of intrusions at follow-up, but not with intrusion-related distress. Therefore, the mediation analyses were repeated controlling for trait rumination when intrusion frequency, but not associated distress, was examined as an outcome variable.

\section{Mediating role of trauma-related rumination in depression-intrusions}

The SPSS macro PROCESS (Hayes, 2013) was used to test the mediating role of trauma-related rumination. 
Table 5 Descriptive statistics and intercorrelations for baseline depression, trait rumination, and trauma-related rumination and intrusion variables

\begin{tabular}{|c|c|c|c|c|c|c|c|c|}
\hline & & $M$ & $S D$ & 1 & 2 & 3 & 4 & 5 \\
\hline 1. & DASS-D & 5.78 & 6.78 & - & & & & \\
\hline 2. & PTQ & 25.32 & 13.08 & $.55 * *$ & - & & & \\
\hline 3. & PTQ-S (day 3) & 16.28 & 12.65 & $.29 * *$ & $.47 * *$ & - & & \\
\hline 4. & PTQ-S (day 7) & 9.71 & 11.44 & .22 & $.41 * *$ & $.79 * *$ & - & \\
\hline 5. & Intrusion frequency (T3) & 2.51 & 3.05 & $.33 * *$ & $.22 *$ & $.47 * *$ & $.32 * *$ & - \\
\hline 6. & Intrusion-related distress (T3) & 2.14 & 1.57 & $.23^{*}$ & .13 & $.53 * *$ & $.51 * *$ & $.46^{* *}$ \\
\hline
\end{tabular}

Note. $N=81$. DASS-D = Depression subscale of the Depression Anxiety Stress Scale; PTQ = Perseverative Thinking Questionnaire; PTQ-S = State version of the Perseverative Thinking Questionnaire.

${ }^{*} p<.05 ;{ }^{* *} p<.01$

Specifically, PROCESS produced estimates of the direct and indirect effects of depression (DASS-D) on intrusion frequency and associated distress through trauma-related rumination (PTQ-S) on day 3. Figure 2 illustrates those pathways with statistical tests of unstandardised regression coefficients $(\beta)$. The bias-corrected bootstrap CIs were based on 5000 samples.

Trauma-related rumination significantly mediated the effect of depression on intrusion frequency (Fig. 2b). That is, the effect of depression was significantly reduced after accounting for trauma-related rumination. This indirect effect represents a medium effect $\left(\boldsymbol{k}^{2}=.12,95 \%\right.$ CI $.03, .24)$ and explained $35 \%$ of the total effect of depression on intrusion frequency (Fig. 2a). Independent of the mechanism of trauma-related rumination, depression remained a significant predictor for intrusion frequency, $t(80)=2.07, p=.04$. Therefore, trauma-related rumination partially mediated the effect of depression on intrusion frequency. In the final model for mediation, $25.7 \%$ of the variance in intrusion frequency was accounted for using the predictors of trauma-related rumination and depression, $F(2,78)=13.47, p<.001$. However, as shown in Fig. 2c, when trait rumination was controlled, trauma-related rumination no longer significantly mediated the relationship between depression and intrusion frequency.

When intrusion-related distress was examined as an outcome variable, trauma-related rumination again significantly mediated the effect of depression (Fig. 2e). That is, the effect of depression was significantly reduced after accounting for trauma-related rumination, which rendered the direct effect of depression to nonsignificance, $t(80)=0.02, p=.38$. This indirect effect represents a medium effect $\left(\boldsymbol{k}^{2}=.15,95 \%\right.$ CI .04, .29), and explained $62 \%$ of the total effect of depression on intrusion-related distress (Fig. 2d). In the final model for mediation, $28.3 \%$ of the variance in intrusion-related distress was accounted for by trauma-related rumination and depression, $F(2$, 78) $=15.41, p<.001$.

\section{DISCUSSION}

This study investigated the effect of depression on trauma intrusions and the role of rumination in the depressionintrusion relationship. As predicted, existing depressive symptoms predicted frequency of trauma intrusions and associated distress following analogue trauma exposure, and trauma-related rumination mediated the depressionintrusion relationship. Not surprisingly, trait ruminative style predicted levels of trauma-related rumination; when trait rumination was controlled, it rendered the mediating role of trauma-related rumination on intrusion frequency to nonsignificance. However, trauma-related rumination was a mediator of intrusion-related distress. Contrary to expectations, experimentally induced low mood did not predict trauma-related rumination or trauma intrusions.

The finding that higher baseline depression symptoms predicted greater numbers of film-related intrusions and associated distress parallels clinical findings that have observed strong relationships between depression and frequency of trauma intrusions (e.g., Brewin et al., 1996) and previous research demonstrating that prior or comorbid depression increases the risk of developing or maintaining PTSD (e.g., Breslau et al., 2000; Shalev et al., 1998). The current findings also lend support to some aspects of emotional processing theory (Foa \& Kozak, 1986), which proposes that depression contributes to the maintenance of posttraumatic stress symptoms. In contrast, in our study, experimentally induced low mood (either prior to or just after the trauma film) did not result in intrusion persistence. This is inconsistent with Wilksch (2011) despite the fact that the current study induced sad mood with the same methods and to a comparable extent (i.e., obtaining a medium effect size). Together, the current finding suggests that in an experimental setting, it is not transient low mood, but naturally occurring depressive symptoms that would prevent habituation of anxiety to trauma memory and cues and thus impede optimal posttrauma adjustment. 
(a)

\begin{tabular}{|c|c|c|}
\hline DASS-D & ctotal entect & Intrusion frequency \\
\hline
\end{tabular}

(b)

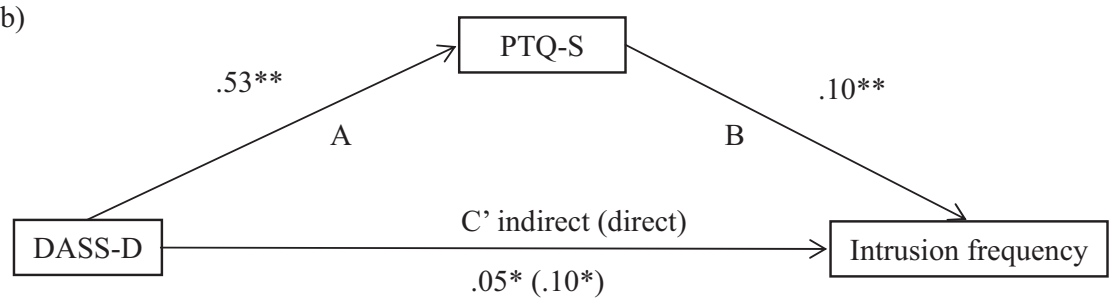

(c)

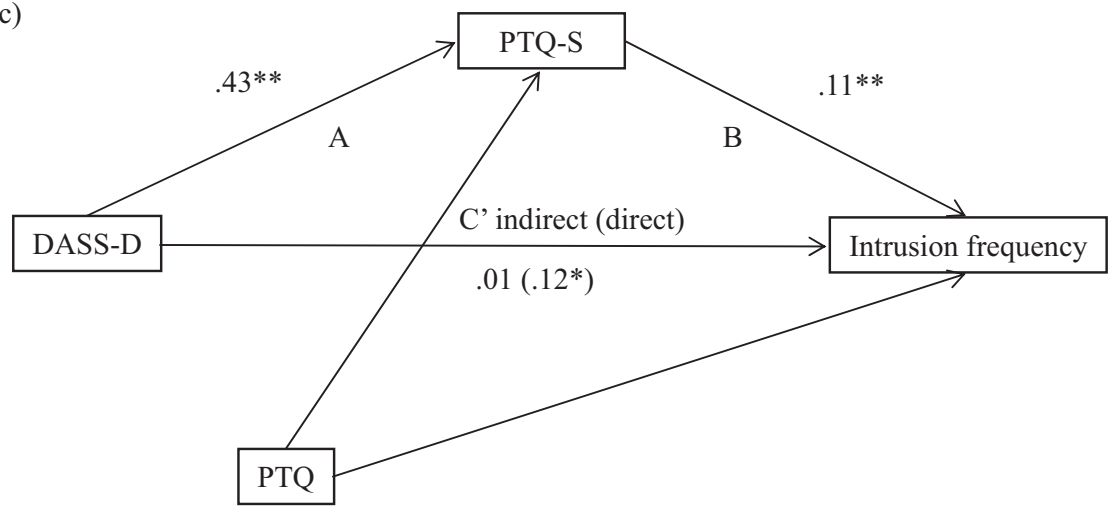

(d)

\begin{tabular}{|c|c|c|}
\hline DASS-D & C total effect & Intrusion-related distress \\
\hline
\end{tabular}

(e)

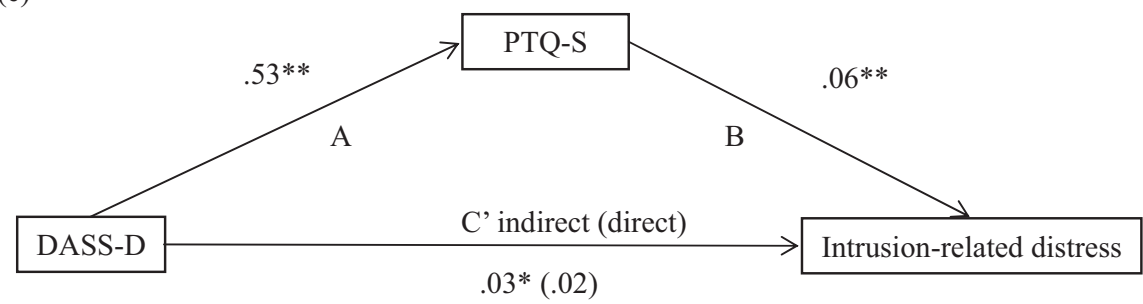

Figure 2 Trauma-related rumination (PTQ-S) as a mediator in the relationship between baseline depression (DASS-D) and trauma intrusion frequency and associated distress, with trait ruminative tendency (PTQ) as a covariate on intrusion frequency. Path values are unstandardised regression coefficients and the associated confidence intervals are shown below. Please see the text for $t$-test values that correspond to these regression coefficients. (a) Depression significantly predicted intrusion frequency $(\beta=.15 ; 95 \%$ CI .05, .24). (b) Trauma-related rumination significantly mediated the effect of depression on intrusion frequency $(\beta=.05 ; 95 \%$ CI .01, .12). The direct effect of depression on intrusion frequency was also significant $(\beta=.10 ; 95 \%$ CI .004, .19). (c) When the effect of trait rumination was controlled, trauma-related rumination no longer significantly mediated the effect of depression on intrusion frequency $(\beta=.01 ; 95 \%$ CI $-.04, .07)$. (d) Depression significantly predicted intrusion-related distress $(\beta=.05 ; 95 \%$ CI .003, .10). (e) Trauma-related rumination significantly mediated the effect of depression on intrusion-related distress $(\beta=.03 ; 95 \%$ CI .01, .07). The direct effect of depression on intrusion-related distress was not significant $(\beta=.02$; $95 \%$ CI $-.03, .07) . N=81 .{ }^{*} p<.05 ; * * p<.01$.

Greater levels of trauma-related rumination were also predicted by higher baseline depression symptom levels. The current finding adds further support for the well-established association between depression and rumination (Ehring $\delta$
Watkins, 2008; Nolen-Hoeksema et al., 2008) in that people with higher depression symptom levels are more likely to employ brooding thinking following a negative event than individuals with lower levels of depression. On the other 
hand, and inconsistent with Hawksley and Davey (2010), temporary low mood was not related to ongoing rumination in the current sample. There are four possible explanations for this: ( 1 ) the smaller effect $(d=0.86$ between the SAF and control groups) of mood induction than that achieved by Hawksley and Davey (2010) $(d=1.31)$; (2) because the effect of sad mood is only relevant when the contents of memory are personal depressive events rather fictional trauma stimuli; (3) because the present study did not have an explicit instruction for participants to continue rumination over the course of follow-up; or (4) because low mood and rumination were not simultaneously induced as done in Hawksley and Davey (2010).

As expected, some evidence was obtained for the relationship between trauma-related rumination and intrusions, that is, levels of rumination during the first 3 days of the film predicted higher numbers of intrusions and corresponding distress levels at the l-week follow-up with a medium effect size. This is consistent with clinical longitudinal research of the effect of ruminative processing on later PTSD symptom severity (e.g., Ehlers et al., 1998; Murray et al., 2002) and experimental research of the effect of induced rumination on subsequent greater intrusions and associated distress levels (Ball \& Brewin, 2012). It is inconsistent with other studies that did not observe this relationship (Ehring, Szheimies, et al., 2009; Zetsche et al., 2009). Interestingly, the present study found the effect of trauma-related rumination in an unselected sample unlike the habitual ruminator sample in Ball and Brewin (2012). Due to the different methodology among these studies and current study, it is difficult to pinpoint possible causes for the discrepancy.

However, the impact of trauma-related rumination on intrusions (both frequency and associated distress) was not observed in later part of the 1 -week interval. In fact, only $n=7-21$ (depending on the day) out of $N=81$ participants reported ruminative thought about the film during day 4 to day 7 (as compared with $n=21-52$ during day 1 to day 3 ), indicating that the analogue trauma stimulus was not strong enough to induce rumination for the entire week in the majority participants. This is somewhat expected given the analogue nature of the trauma stimulus. Despite this, intrusive memories returned to participants when they returned for the 1-week follow-up session, which might have functioned as a reminder of the film. It is important to note that rumination in the days following the film predicted the number of intrusions and related distress even after 1 week had elapsed. Clinically, this would imply that there may be an important window for early intervention to address rumination (e.g., within 3 days of trauma exposure in the present study) that might assist in preventing later suboptimal habituation of anxiety to trauma memory and reminders. Clearly, future research would need to for- mally test this with participants who are dysphoric or clinically depressed, and following real trauma.

As expected, some evidence was found that traumarelated rumination is one mechanism by which depression contributes to the levels of trauma intrusions and associated distress. Thus, it is possible that for actual trauma survivors with depression, trauma-related rumination may increase the risk of chronic PTSD. However, the mediating role of trauma-related rumination on intrusion frequency was rendered nonsignificant when trait rumination was controlled. This is in contrast to a nonsignificant relationship between trait rumination and intrusion-related distress. Hence, trait ruminative tendency may determine the degree of rumination following trauma exposure which in turn increases intrusions, but the distress caused by intrusions seems somewhat independent of trait rumination processes. Despite this, however, trait rumination was found to positively correlate with depression and traumarelated rumination, and rumination is thought to increase depression symptoms (e.g., Nolen-Hoeksema et al., 2008). Therefore, the role of trait ruminative tendency in PTSD symptomatology in comorbid depression should continue to be examined.

Further, it was interesting that while there was a partial mediating role of trauma-related rumination when frequency of intrusions was examined, a full mediation was observed with the associated distress accompanying such intrusions. That is, compared with people with lower levels of depression, while individuals with higher levels of depression might experience more frequent trauma intrusions independent of the mechanism of trauma-related rumination, they may experience greater distress from those intrusions through ruminative processing of trauma. Given that it seems it is the distress provoked by trauma intrusions that plays a key role in predicting the maintenance of PTSD, not intrusion frequency per se (Steil \& Ehlers, 2000), traumarelated rumination appears to be an important target for intervention for PTSD with comorbid depression.

Overall, the current pattern of results appear to suggest that it was not temporarily induced low mood but naturally occurring symptoms of depression (which was associated with trait ruminative tendency) that contributed to traumarelated rumination, resulting in the trauma intrusions and associated distress. Accordingly, emotional processing theory (Foa \& Kozak, 1986) would benefit from explicitly considering rumination to be a critical factor in the process of adjustment following trauma. Ehlers and Clark (2000) have already proposed rumination to be a dysfunctional cognitive strategy that leads to the persistence of PTSD, and have suggested specific unhelpful characteristics of rumination (i.e., avoidance, enhancing negative appraisals, and providing increased access to trauma memory). An empirical examination of these proposed functions of trauma-related 
rumination in trauma victims with high levels of depression would assist in developing a more sophisticated explanation of how pre-existing psychopathology or processes such as depression and rumination drive the maintenance of intrusions following trauma exposure.

Several limitations are acknowledged. First, while analogue trauma films are useful in providing the means of studying the impact of cognitive processes such as rumination on intrusions (Holmes \& Bourne, 2008), watching a trauma film is a voluntary exercise, and not the same as experiencing a real-life event. Second, the filler task used following the mood induction for the SBF group might have functioned as a distraction, impacting on cognitive processing of trauma, which might in turn have affected later intrusions and rumination. Although it should be noted, this group demonstrated a similar pattern of findings as the SAF condition (which was not given the filler task). Third, the majority of participants (approximately $80 \%$ ) reported levels of depressive symptoms within normal limits at baseline, which might be due to the participation criteria that excluded people with a formal diagnosis of depression. Whether stronger effects of a mood induction and consequently a greater mediating role of rumination would have been observed in participants with higher levels of depressed symptoms remain to be seen.

The current study found that existing depressive symptoms, but not momentary low mood, contributes to the experience of distressing analogue trauma intrusions and provides preliminary evidence for the mediating role of rumination in the depression-intrusion association. Future research needs to examine the exact mechanisms through which posttrauma rumination contributes to anxiety to trauma memory and reminders, as this would in turn inform how to best assist optimal recovery following trauma, which is particularly relevant in PTSD sufferers with comorbid depression.

\section{REFERENCES}

American Psychiatric Association. (2013). Diagnostic and statistical manual of mental disorders. (5th ed.). Arlington, VA: American Psychiatric Publishing.

Ball, S., \& Brewin, C. (2012). The effect of rumination on intrusive images and mood: An experimental investigation using the trauma film paradigm. Journal of Experimental Psychopathology, 3(2), 297-309. doi:10.5127/jep.019511

Birrer, E., Michael, T., \& Munsch, S. (2007). Intrusive images in PTSD and in traumatised and non-traumatised depressed patients: A cross-sectional clinical study. Behaviour Research and Therapy, 45(9), 2053-2065. doi:10.1016/j.brat.2007.03.005

Blanchard, E. B., Buckley, T. C., Hickling, E. J., \& Taylor, A. E. (1998). Posttraumatic stress disorder and comorbid major depression: Is the correlation an illusion? Journal of Anxiety Disorders, $12(1), 21-37$.
Breslau, N., Davis, G. C., Peterson, E. L., \& Schultz, L. (1997). Psychiatric sequelae of posttraumatic stress disorder in women. Archives of General Psychiatry, 54(1), 81-87.

Breslau, N., Davis, G. C., Peterson, E. L., \& Schultz, L. R. (2000). A second look at comorbidity in victims of trauma: The posttraumatic stress disorder-major depression connection. Biological Psychiatry, 48(9), 902-909. doi:10.1016/S0006-3223(00) 00933-1

Brewin, C. R., Dalgleish, T., \& Joseph, S. (1996). A dual representation theory of posttraumatic stress disorder. Psychological Review, 103(4), 670-686.

Brewin, C. R., Hunter, E., Carroll, F., \& Tata, P. (1996). Intrusive memories in depression: An index of schema activation? Psychological Medicine, 26(6), 1271-1276.

Brown, T. A., Chorpita, B. F., Korotitsch, W., \& Barlow, D. H. (1997). Psychometric properties of the Depression Anxiety Stress Scales (DASS) in clinical samples. Behaviour Research and Therapy, 35(1), 79-89. doi:10.1016/S0005-7967(96)00068-X

Ehlers, A., \& Clark, D. M. (2000). A cognitive model of posttraumatic stress disorder. Behaviour Research and Therapy, 38(4), 319-345.

Ehlers, A., \& Steil, R. (1995). Maintenance of intrusive memories in posttraumatic stress disorder: A cognitive approach. Behavioural and Cognitive Psychotherapy, 23(3), 217-249. doi:10.1017/ S135246580001585X

Ehlers, A., Mayou, R. A., \& Bryant, B. (1998). Psychological predictors of chronic posttraumatic stress disorder after motor vehicle accidents. Journal of Abnormal Psychology, 107(3), 508-519. doi:10.1037/0021-843X.107.3.508

Ehring, T. (2007). Development and validation of a content-independent measure of perseverative thinking. Paper presented at the World Congress of Behavioural and Cognitive Therapies, Barcelona.

Ehring, T., \& Watkins, E. R. (2008). Repetitive negative thinking as a transdiagnostic process. International journal of cognitive therapy, 1(3), 192-205. doi:10.1680/ijct.2008.1.3.192

Ehring, T., Fuchs, N., \& Klasener, I. (2009). The effects of experimentally induced rumination versus distraction on analogue posttraumatic stress symptoms. Behavior Therapy, 40(4), 403-413. doi:10.1016/j.beth.2008.10.001

Ehring, T., Szeimies, A. K., \& Schaffrick, C. (2009). An experimental analogue study into the role of abstract thinking in traumarelated rumination. Behaviour Research and Therapy, 47(4), 285293. doi:10.1016/j.brat.2008.12.011

Ehring, T., Zetsche, U., Weidacker, K., Wahl, K., Schonfeld, S., \& Ehlers, A. (2011). The Perseverative Thinking Questionnaire (PTQ): Validation of a content-independent measure of repetitive negative thinking. Journal of Behavior Therapy and Experimental Psychiatry, 42(2), 225-232. doi:10.1016/j.jbtep.2010.12.003

Eich, E., Ng, J. W., Macaulay, D., Percy, A. D., \& Grebneva, I. (2007). Combining music with thought to change mood. In J. A. Coan $\delta$ J. B. Allen (Eds.), The handbook of emotion elicitation and assessment (pp. 124-136). New York: Oxford University Press.

Evans, C., Ehlers, A., Mezey, G., \& Clark, D. M. (2007). Intrusive memories in perpetrators of violent crime: Emotions and cognitions. Journal of Consulting and Clinical Psychology, 75(1), 134-144. doi:10.1037/0022-006X.75.1.134

Foa, E. B., \& Kozak, M. J. (1986). Emotional processing of fear: Exposure to corrective information. Psychological Bulletin, 99(1), 20-35.

Foa, E. B., Ehlers, A., Clark, D. M., Tolin, D. F., \& Orsillo, S. M. (1999). The Posttraumatic Cognitions Inventory (PTCI): Development and validation. Psychological Assessment, 11(3), 303-314. doi:10.1037/1040-3590.11.3.303

Hawksley, J., \& Davey, G. C. (2010). Mood-as-input and depressive rumination. Behaviour Research and Therapy, 48(2), 134-140. doi:10.1016/j.brat.2009.10.004 
Hayes, A. F. (2013). Introduction to mediation, moderation, and conditional process analysis: A regression-based approach. New York, NY: Guilford Press.

Holmes, E. A., \& Bourne, C. (2008). Inducing and modulating intrusive emotional memories: A review of the trauma film paradigm. Acta Psychologica, 127(3), 553-566. doi:10.1016/j.actpsy.2007.11 .002

Joormann, J., Dkane, M., \& Gotlib, I. H. (2006). Adaptive and maladaptive components of rumination? Diagnostic specificity and relation to depressive biases. Behavior Therapy, 37(3), 269280. doi:10.1016/j.beth.2006.01.002

Kessler, R. C., Sonnega, A., Bromet, E., Hughes, M., \& Nelson, C. B. (1995). Posttraumatic stress disorder in the National Comorbidity Survey. Archives of General Psychiatry, 52(12), 1048-1060.

Kuyken, W., \& Brewin, C. R. (1994). Intrusive memories of childhood abuse during depressive episodes. Behaviour Research and Therapy, 32(5), 525-528.

Lovibond, S. H., \& Lovibond, P. F. (1995). Manual for the depression anxiety stress scales [kit]. Sydney, NSW: Psychology Foundation of Australia.

Martin, L. L., \& Tesser, A. (1996). Some ruminative thoughts. In R. S. Wyer (Ed.), Advances in social cognition. Vol. 9. Mahwah, NJ: Lawrence Erlbaum.

Meeten, F., \& Davey, G. C. (2011). Mood-as-input hypothesis and perseverative psychopathologies. Clinical Psychology Review, 31(8), 1259-1275. doi:10.1016/j.cpr.2011.08.002

Michael, T., Halligan, S. L., Clark, D. M., \& Ehlers, A. (2007). Rumination in posttraumatic stress disorder. Depression and Anxiety, 24(5), 307-317. doi:10.1002/da.20228

Murray, J., Ehlers, A., \& Mayou, R. A. (2002). Dissociation and post-traumatic stress disorder: Two prospective studies of road traffic accident survivors. The British Journal of Psychiatry: The Journal of Mental Science, 180(4), 363-368. doi:10.1192/ bjp.180.4.363

Nixon, R. D., Resick, P. A., \& Nishith, P. (2004). An exploration of comorbid depression among female victims of intimate partner violence with posttraumatic stress disorder. Journal of Affective Disorders, 82(2), 315-320. doi:10.1016/j.jad.2004.01.008
Nixon, R. D., Nehmy, T., \& Seymour, M. (2007). The effect of cognitive load and hyperarousal on negative intrusive memories. Behaviour Research and Therapy, 45(11), 2652-2663. doi:10.1016/ j.brat.2007.06.010

Nolen-Hoeksema, S. (1991). Responses to depression and their effects on the duration of depressive episodes. Journal of Abnormal Psychology, 100(4), 569-582.

Nolen-Hoeksema, S., Wisco, B. E., \& Lyubomirsky, S. (2008). Rethinking rumination. Perspectives on Psychological Science, 3(5), 400-424. doi:10.1111/j.1745-6924.2008.00088.x

Shalev, A. Y., Freedman, S., Peri, T., Brandes, D., Sahar, T., Orr, S. P., \& Pitman, R. K. (1998). Prospective study of posttraumatic stress disorder and depression following trauma. The American Journal of Psychiatry, 155(5), 630-637.

Steil, R., \& Ehlers, A. (2000). Dysfunctional meaning of posttraumatic intrusions in chronic PTSD. Behaviour Research and Therapy, 38(6), 537-558.

Watkins, E. R. (2008). Constructive and unconstructive repetitive thought. Psychological Bulletin, 134(2), 163-206. doi:10.1037/ 0033-2909.134.2.163

Weathers, F. W., Litz, B. T., Herman, D. S., Huska, J. A., \& Keane, T. M. (1993). The PTSD Checklist (PCL): Reliability, validity, and diag nostic utility. Paper presented at the 9th Annual Conference of the ISTSS, San Antonio.

Werner-Seidler, A., \& Moulds, M. L. (2011). Autobiographical memory characteristics in depression vulnerability: Formerly depressed individuals recall less vivid positive memories. Cognition and Emotion, 25(6), 1087-1103. doi:10.1080/02699931.2010 .531007

Wilksch, S. (2011). Emotional processing in Posttraumatic Stress Disorder (PTSD): Improving the outcomes following Cognitive Behaviour Therapy (CBT) for PTSD. Flinders University.

Zetsche, U., Ehring, T., \& Ehlers, A. (2009). The effects of rumination on mood and intrusive memories after exposure to traumatic material: An experimental study. Journal of Behavior Therapy and Experimental Psychiatry, 40(4), 499-514. doi:10.1016/j.jbtep.2009 .07 .001 